\title{
Corrigendum
}

\section{Patterns in weight reduction behaviour by weight status in schoolchildren - Corrigendum}

\author{
Colette Kelly, Michal Molcho and Saoirse Nic Gabhainn
}

doi:10.1017/S1368980009992102, Published online by Cambridge University Press 27 November 2009

First published online 3 September 2010

In table 1, p 1231 the figures in columns 1 and 2 should read as follows:

Table 1 Sociodemographic characteristics of respondents by weight reduction behaviour (dieting) and weight status

\begin{tabular}{|c|c|c|c|c|c|c|}
\hline & \multicolumn{2}{|c|}{ All } & \multicolumn{2}{|c|}{ Non-overweight } & \multicolumn{2}{|c|}{ Overweight and obese } \\
\hline & $\begin{array}{l}\text { Dieting } \\
(n \text { 455) }\end{array}$ & $\begin{array}{l}\text { Not dieting } \\
(n 3144)\end{array}$ & $\begin{array}{l}\text { Dieting } \\
(n \text { 319) }\end{array}$ & $\begin{array}{l}\text { Not dieting } \\
(n \text { 2777) }\end{array}$ & $\begin{array}{l}\text { Dieting } \\
(n \text { 136) }\end{array}$ & $\begin{array}{l}\text { Not dieting } \\
\quad(n 367)\end{array}$ \\
\hline \multicolumn{7}{|l|}{ Age (years) } \\
\hline Mean & $14 \cdot 8$ & $14 \cdot 5$ & $14 \cdot 9$ & $14 \cdot 5$ & $14 \cdot 7$ & $14 \cdot 5$ \\
\hline SD & $1 \cdot 44$ & 1.56 & $1 \cdot 41$ & 1.56 & 1.50 & $1 \cdot 62$ \\
\hline Male (\%) & $40 \cdot 4$ & $59 \cdot 2$ & $35 \cdot 7$ & $58 \cdot 4$ & $51 \cdot 5$ & $65 \cdot 1$ \\
\hline Female (\%) & $59 \cdot 6$ & $40 \cdot 8$ & $64 \cdot 3$ & $41 \cdot 6$ & $48 \cdot 5$ & 34.9 \\
\hline Age $10-11$ yr (\%) & $5 \cdot 1$ & $7 \cdot 9$ & $4 \cdot 7$ & $7 \cdot 6$ & $5 \cdot 9$ & $10 \cdot 4$ \\
\hline Age $12-14$ yr (\%) & $43 \cdot 3$ & $46 \cdot 6$ & $40 \cdot 8$ & $46 \cdot 8$ & $49 \cdot 3$ & $45 \cdot 2$ \\
\hline Age $15-17$ yr (\%) & $51 \cdot 6$ & $45 \cdot 5$ & $54 \cdot 5$ & $45 \cdot 6$ & $44 \cdot 9$ & $44 \cdot 4$ \\
\hline High social class $(\%)$ & 33.6 & $30 \cdot 9$ & $41 \cdot 4$ & $34 \cdot 8$ & $23 \cdot 8$ & $29 \cdot 1$ \\
\hline Medium social class (\%) & $42 \cdot 2$ & $40 \cdot 4$ & $41 \cdot 8$ & 43.9 & 54 & $50 \cdot 3$ \\
\hline Low social class (\%) & $17 \cdot 1$ & $19 \cdot 2$ & $16 \cdot 8$ & $21 \cdot 3$ & $22 \cdot 2$ & $20 \cdot 6$ \\
\hline
\end{tabular}

\section{Reference}

Kelly C, Molcho M \& Nic Gabhainn S (2010) Weight reduction behaviour by weight status in schoolchildren. Public Health Nutr 13, 1229-1236. 